\title{
Histomorphological responses after therapy with pegylated interferon a-2a in patients with essential thrombocythemia (ET) and polycythemia vera (PV)
}

Lucia Masarova', C. Cameron Yin², Jorge E. Cortes ${ }^{1}$, Marina Konopleva', Gautam Borthakur ${ }^{1}$, Kate J. Newberry ${ }^{1}$, Hagop M. Kantarjian ${ }^{1}$, Carlos E. Bueso-Ramos² and Srdan Verstovsek ${ }^{1^{*}}$

\begin{abstract}
Background: Pegylated interferon alfa-2a (PEG-IFN- $a-2 a$ ) is a potent immunomodulating agent capable of inducing high rate of hematologic and even complete molecular remission in patients with essential thrombocythemia (ET) and polycythemia vera (PV). We recently reported results of a phase 2 trial of PEG-IFN-a-2a in 83 patients with ET and PV after a median follow-up of 83 months. Here we report an analysis of bone marrow (BM) responses in these patients.

Methods: Among 83 patients, 58 (70\%, PV 25, ET 31) had evaluable BM samples. BM responses and fibrosis grading were defined according to the International Working Group for Myeloproliferative Neoplasms Research and Treatment, and the European Consensus on grading of BM fibrosis, respectively. BM was assessed prior to enrollment, and every 6-24 months while on therapy in all patients, and after therapy discontinuation in some patients.

Results: The median age of analyzed 58 patients was 52 years, and 29\% were males. After a median follow-up of 84 months, 32 patients are still on study. Hematologic (HR) and molecular responses (MR) were seen in 93 and $69 \%$ patients, respectively. Twenty-nine patients (50\%) had a BM response, including 13 (22\%) with a complete BM response (BM-CR). Moreover, 13 patients (22\%) have experienced complete resolution of bone marrow reticulin fibrosis. Patients with BM response had higher duration of HR and MR, and lower discontinuation rate. Furthermore, patients with BM-CR had a higher probability of complete MR. The median duration of BM-CR was 30 months, and 9 patients have maintained their BM-CR (69\%), including five who have maintained their response after discontinuation of therapy. Despite this observation, the pattern of HR, MR and BM response, their durability and interrelation was heterogeneous.
\end{abstract}

Conclusions: Our results show the ability of PEG-IFN-a-2a to induce complete BM responses in a subset of ET and PV patients, but its correlation with durable clinically relevant treatment benefit warrants further investigation.

Trial registration This study is registered with ClinicalTrials.gov (NCT00452023), and is ongoing but not enrolling new patients.

Keywords: Essential thrombocythemia, Polycythemia vera, Pegylated interferon alfa-2a, Histomorphological response

\footnotetext{
*Correspondence: sverstov@mdanderson.org

${ }^{1}$ Department of Leukemia, The University of Texas MD Anderson Cancer

Center, 1515 Holcombe Blvd., Unit 428, Houston, TX 77030, USA

Full list of author information is available at the end of the article
} 


\section{Background}

Essential thrombocythemia (ET) and polycythemia vera (PV) are BCR-ABL-negative myeloproliferative neoplasms (MPN) characterized by an overproduction of mature blood elements, tendencies toward thrombosis and hemorrhage, extramedullary hematopoiesis (mild splenomegaly), and transformation to overt myelofibrosis (MF)/acute myeloid leukemia (AML) [1,2]. The therapeutic approach for patients at high risk of thrombosis include cytoreductive therapy such as hydroxyurea [3] or recombinant interferon-alfa [4-6]. Several clinical studies have reported that pegylated interferon-alfa can induce complete hematologic remission (CHR) in majority of patients with ET and $\mathrm{PV}$, and is even capable to induce complete molecular remission $(C M R)$ in some patients [6-8]. Similarly, clinical benefit and high HR were noticed in patients with early stages of MF, where interferon-alfa has been shown to possibly reverse bone marrow (BM) fibrosis $[9,10]$.

Recently, we reported a long-term follow-up of a prospective, phase 2 clinical trial of pegylated interferon alfa-2a (PEG-IFN- $\alpha-2 a)$ in 83 patients with PV or ET. After a median follow-up of 83 months, overall HR and MR were noticed in 80 and 63\%, respectively, and were shown durable in some patients, with overall median response durations of 66 and 53 months, respectively [8]. Our findings confirmed observations by others that neither baseline molecular status nor achievement of a MR on therapy, are a prerequisite for achieving a $H R$, and some patients continued to derive significant clinical benefit despite losing their HR or best MR. Histomorphologic evaluation of BM is an important part of establishing a diagnosis [11] and in response assessment in patients with ET/PV [12]. According to the most recent WHO classification, a major criterion for diagnosis of ET is a BM biopsy showing proliferation of the megakaryocyte lineage, with no significant increase in granulopoiesis or erythropoiesis, and very rarely minor (grade 1) increase in reticulin fibers; and for PV, a BM biopsy showing hypercellularity with panmyelosis [11]. The development of BM fibrosis is considered part of the natural evolution of these diseases, and has been reported in up to 20 and $51 \%$ of patients with ET and PV during their disease duration, respectively [13-15]. Although there is an increasing evidence of direct correlation between a higher BM fibrosis grade in patients with ET/PV and signs of progressive disease, such as splenomegaly, cytogenetic aberrations, increased risks of vascular complications, and transformation to overt MF [14-17], the prognostic value of improvement in BM fibrosis (or other BM characteristics) in patients with PV and ET while on therapy, remains unknown. Despite promising preclinical data showing that the robust immunomodulatory and anti-proliferative effects of interferon-alfa may reverse
BM fibrosis via its stimulatory effects on hematopoietic stem cells and inhibitory effects on megakaryopoiesis [18-20], morphologic remission with interferon-alfa therapy has been rarely reported [21-23]. Herein, we report the histomorphological BM responses of patients with ET/PV treated with PEG-IFN- $\alpha-2 a$ in a prospective, open-label, single-center, phase 2 clinical trial.

\section{Patients and methods}

Overall, 83 patients (43 PV, 40 ET) were enrolled in a clinical trial of PEG-IFN- $\alpha$-2a between 2005 and 2009 at our institution. Detailed inclusion and exclusion criteria, treatment schedule, response assessment, statistical considerations and primary analyses have been previously reported [6, 24].

Hematologic and molecular responses, as defined by the European LeukemiaNet [12], were assessed every 3 and 6 months, respectively. Histomorphology of the BM, including fibrosis grading, and morphological responses according to European LeukemiaNet, International Working Group-Myeloproliferative Neoplasms Research and Treatment (IWG-MRT) and European Consensus $[12,25]$ were assessed by expert hematopathologists at our institution before study enrollment, every 6-12 months during the first 3 years while on study, and then every 12-24 months during the subsequent years. Some patients continued to have BM evaluated every 12-24 months after treatment discontinuation. Complete BM remission (BM-CR) was defined by ELN and IWGMRT criteria [12] as follows: absence of more than grade 1 reticulin fibrosis and disappearance of megakaryocyte hyperplasia in ET or trilinear hyperplasia with ageadjusted normocellularity in PV. An incomplete, partial response (BM-PR) was defined as fibrosis grading that had improved by at least one grade level on at least 2 consecutive samples taken $\geq 12$ months apart, but with persistent morphological features of ET or PV.

Responses and clinical data were analyzed using descriptive statistics. Fisher's exact and Wilcoxon ranksum tests were used to compare categorical and continuous variables, respectively. Time to BM-responses and its duration was estimated by using Kaplan-Meier method. GraphPad Prism and SPSS v.23 were used for all analyses.

\section{Results}

Among 83 patients enrolled to this study, 58 (70\%) had BM samples that were evaluable for BM response assessment. Eighteen patients treated for $\leq 12$ months and 7 without consecutive BM samples were not evaluable for BM response. Demographics and clinical characteristics of 58 currently analyzed patients are detailed in Table 1. The median follow-up on study was 84 months (range 36-107), and median number of BM 
Table 1 Clinical and demographic characteristics of all patients, and stratified by BM response

\begin{tabular}{|c|c|c|c|c|}
\hline Characteristics & All pts, $n=58$ & BM-NR, n = 29 & BM-PR, n = 16 & $\mathrm{BM}-\mathrm{CR}, \mathrm{n}=13$ \\
\hline PV/ET, no. & $27 / 31$ & $15 / 14$ & $5 / 11$ & $7 / 6$ \\
\hline Median age, years (range) & $52(19-75)$ & $53(19-72)$ & $55(33-70)$ & $52(23-75)$ \\
\hline JAK2 V617F-positive patients, no (\%) & $42(72)$ & $22(76)$ & $9(56)$ & $11(84)$ \\
\hline Other driver mutations, no (\%) & CALR 6, MPL 2, TN 8 & CALR 1, TN 6 & CALR 5, MPL 2 & TN 2 \\
\hline JAK2 V617F allele burden, median (range) & $34(3-89)$ & $32.4(3-82)$ & $41(18-89)$ & $30(5-76)$ \\
\hline Abnormal karyotype, no & 3 & - & 2 & 1 \\
\hline Median white blood cell count, 109/L (range) & $9(4-19)$ & $9(4-19)$ & $9(3-19)$ & $8(4-12)$ \\
\hline Median hemoglobin, g/dL (range) & $13(9-19)$ & $14(12-17)$ & $13(9-18)$ & $13(12-19)$ \\
\hline Median platelet count, $10^{9} / \mathrm{L}$ (range) & $721(128-1646)$ & $629(185-1646)$ & $866(128-1423)$ & $420(236-1020)^{*}$ \\
\hline Significant splenomegaly, n (\% of known) & $9 / 55(16)$ & $5 / 28(18)$ & $3 / 15(20)$ & $1 / 12(8)$ \\
\hline Disease-related symptoms, n (\%) & $30(52)$ & $17(59)$ & $6(38)$ & $7(54)$ \\
\hline No. of previously treated (\%) & $36(62)$ & $16(55)$ & $11(69)$ & $9(69)$ \\
\hline
\end{tabular}

* $p$ value $<0.05$

a Significant splenomegaly defined as a palpable spleen $>5 \mathrm{~cm}$ below costal margin (BCM), Abnormal karyotype: BM-PR: DER (13;22)(Q10;Q10) $(n=1),+$ MAR $(n=1)$, BM-CR: +1, DER $(1 ; 22)(Q 10 ; Q 10)(n=1)$

samples per patient was 8 (range 3-12). Median age was 52 years (range $19-75)$ and $29 \%(n=17)$ were men. Sixty-two percent of patients $(n=36)$ were previously treated, the majority of them with hydroxyurea ( $n=27$, $75 \%)$. Except for abnormalities in marrow cellularity, atypical megakaryocytes characteristic of ET/PV [11], 35 patients (25 PV, $10 \mathrm{ET}$; 60\%) had reticulin fibrosis of grade MF-1 or MF- 2 according to European grading [25]. Median exposure to PEG-IFN- $\alpha-2$ a was 80 months (range 15-107). The starting doses of PEG-IFN- $\alpha-2 \mathrm{a}$ given subcutaneously in a weekly schedule were $450 \mu \mathrm{g}$ in 2 patients, $360 \mu \mathrm{g}$ in 2 patients, $270 \mu \mathrm{g}$ in 11 patients, $180 \mu \mathrm{g}$ in 20 patients and $90 \mu \mathrm{g}$ in 35 patients. The dose was modified throughout the study based on toxicity or lack of efficacy. At the time of this report, out of 58 patients, $32(55 \%)$ are still on study, 24 (75\%) of whom are actively being treated.

\section{Hematologic response}

Initial HR was achieved in 54 (93\%) patients, and it was sustained in $33 \%$ of them $(n=18)$ throughout the entire follow-up on a study with a median response duration of 92 months (range 58-101+). The remaining 36 patients lost their HR after a median duration of 55 months (range 10.4-91). Sixteen patients who lost their HR, are still on study, 14 of them actively treated, as they continue to derive clinical benefit (no clinical symptoms and organomegaly) with good tolerance.

\section{Molecular response}

Among 44 patients who were JAK2V617F-positive, the MR rate was $72 \%$ ( $n=32$; a complete MR [CMR; undetectable JAK2V617F allele burden] in 9, a partial MR [PMR; 50\% reduction in JAK2V617F allele burden] in 19, and a minor MR [mMR; 20-49\% reduction in JAK2V617F allele burden] in 4 patients, Table 2). The median JAK2V617F allele burden was $25 \%$ at baseline for those achieving CMR, while it decreased from 41 to $8 \%$ in those with PMR, and $34-24 \%$ in those with mMR. Pre-treatment allele burden did not differ among patients with CMR, PMR and mMR. The response duration was similar in patients with CMR (68 months; range 21-94), PMR (53 months; range 7-79) and mMR (50 months; range 12-60).

All patients with CMR (100\%), 4 patients with PMR (21\%) and 2 with mMR (50\%) have maintained their best MR throughout the entire follow-up. Overall 14 patients with PMR (74\%) lost their PMR (7 completely and 7 has maintained a mMR). One patient with a PMR received an allogeneic stem cell transplantation for concurrent large B cell lymphoma and was therefore not evaluable. The median JAK2 allele burden at baseline and at the time of best response did not differ between those who maintained a PMR and those who did not. All but one patients were actively treated at the time of their PMR loss (drug was withheld in one patient for 20 months due to G1 adverse events when he lost his PMR).

\section{Bone marrow response}

Overall, 29 patients (50\%) had BM response, including 13 patients (22\%) with BM-CR (Table 3, Fig. 1 ), and 16 patients (28\%) with BM-PR [12]. Furthermore, 13 patients (22\%) had complete resolution of bone marrow reticulin fibrosis (MF-0), including 3 patients with initial grade 2 . Demographic and clinical characteristics and MR patterns in all evaluable patients $(n=58)$ stratified by BM response (BM-CR, BM-PR, and BM-NR) are shown in 
Tables 1 and 2. Except for increased platelets at baseline in those with BM-PR $(\mathrm{p}<0.001)$, likely due to the higher proportion of ET patients in that group, no other differences in basic demographic or clinical parameters were present among different response groups (Table 1). There was no difference in the median time to achievement of a best $\mathrm{BM}$ response or its duration among patients with BM-CR and BM-PR (Table 2, Fig. 2a-f). Nine (69\%) patients with BM-CR and 14 (88\%) patients with BM-PR have sustained their best $B M$ response.

The median initial dose of PEG-IFN- $\alpha-2 \mathrm{a}$ was $180 \mu \mathrm{g}$ per week (range 90-450 mcg) in each group. All patients required dose adjustments either for toxicity $(n=44)$ or for lack of efficacy $(n=14)$. Median cumulative weekly dose of PEG-IFN- $\alpha-2 \mathrm{a}$ until best BM response was $45 \mu \mathrm{g}$ per week, similar for patients with BM-CR and BM-PR. No differences in median weekly dose of PEG-IFN- $\alpha$-2a while on therapy or overall treatment duration were found among patients with BM-CR, BM-PR and BM-NR.

Interestingly, although the overall duration of therapy was similar among groups (Fig. 2d-f), significantly fewer patients with BM-NR remained on the therapy at the time of last follow-up than those with BM-CR or BM-PR ( $35 \%$ vs $69 \%$ vs $81 \%, p=0.03$ ). Patients with a BM-CR had a significantly longer $\mathrm{HR}$ duration than patients with BM-PR and BM-NR (87 vs 69 vs 50 months; $\mathrm{p}=0.01$ ), respectively. However, no difference in rate of the initial HR was seen among groups.
Patients with a BM response (BM-CR \& BM-PR) had a higher initial MR rate than patients with BM-NR (91 and $89 \%$ vs $50 \%, p=0.04)$. Furthermore, patients with a BM-CR had higher CMR rate than all other patients (BM-PR \& BM-NR) $(70 \%$ vs $12 \%$; p < 0.001; Table 2). Similarly, patients with PV who have achieved CMR have experienced significant reduction in bone marrow cellularity $(80-40 \%, \mathrm{p}=0.02)$ when compared to the remaining PV patients. On the other hand, durability of MR was the same regardless of BM response status. Despite the lower MR rate in patients with BM-NR, we have still observed CMR and PMR in 2 and 11 of these patients, respectively.

Probability of maintaining achieved BM response was 92 and $40 \%$ at 2 and 4 years for BM-CR, and 96 and $62 \%$ for all BM-responses, respectively (Fig. 2g-i). Probability of achieving any BM response and BM-CR on a study in all patients treated with PEG-INF- $\alpha-2$ a was 26 and $9 \%$ at 4 years, and 61 and $27 \%$ at 7 years, respectively (Fig. 3a, b).

Correlation between HR, MR and BM response in patients with BM-CR are individually detailed in Figs. 4, 5 and 6. All 13 patients with BM-CR have initially achieved HR, which was sustained in 9 patients at the time of last follow-up. Two of those 4 patients who has lost their HR, remained in sustained BM-CR. Seven and 3 of these patients achieved CMR and PMR, respectively. None of the patients with CMR has experienced a

Table 2 Responses details, stratification by BM response

\begin{tabular}{|c|c|c|c|c|}
\hline Characteristics & All patients, $n=58$ & BM-NR, n = 29 & BM-PR, $n=16$ & $B M-C R, n=13$ \\
\hline Initial HR, no. (\%) & $54(93)$ & $26(90)$ & $15(94)$ & $13(100)$ \\
\hline Last HR, no (\% of initial HR) & $22(41)$ & $7(27)$ & $5(33)$ & $9(69)$ \\
\hline Duration of HR, months (range) & $90(10-101)$ & $50(10-98)$ & $69(17-96)$ & $87(59-101)^{*}$ \\
\hline Initial MR, no. (\% of JAK2+), type & $\begin{array}{l}33 / 44(75) \\
9 \text { CMR, } 20 \text { PMR, } 4 \text { mMR }\end{array}$ & $\begin{array}{l}15(68) \\
2 \text { CMR, } 11 \text { PMR, } 2 \text { mMR }\end{array}$ & $\begin{array}{l}8(89) \\
6 \text { PMR, } 2 \text { mMR }\end{array}$ & $\begin{array}{l}10(91) \\
7 \mathrm{CMR}^{*}, 3 \mathrm{PMR}\end{array}$ \\
\hline Last MR, no (\% of initial overall MR), type & $\begin{array}{l}22 \text { (67) } \\
9 \text { CMR; } 4 \text { PMR, } 9 \text { mMR }\end{array}$ & $\begin{array}{l}7(47)^{\mathrm{a}} \\
2 \mathrm{CMR}, 5 \mathrm{mMR}\end{array}$ & $\begin{array}{l}6(75) \\
3 \text { PMR, } 3 \text { mMR }\end{array}$ & $\begin{array}{l}9(90) \\
7 \text { CMR, } 1 \text { PMR, } 1 \text { mMR }\end{array}$ \\
\hline Time to BM response, months (range) & $52(12-72)$ & NA & $54(12-72)$ & $52(30-72)^{b}$ \\
\hline BM response duration, months (range) & $36(12-66)$ & NA & $36(12-66)$ & $30(24-52)$ \\
\hline Last $B M$ response, no (\% of initial), type & 9 BM-CR; 14 BM-PR & NA & $\begin{array}{l}14 \mathrm{BM}-\mathrm{PR}(88) \\
2 \mathrm{BM}-\mathrm{NR}(12)\end{array}$ & $\begin{array}{l}9 \mathrm{BM}-\mathrm{CR}(69) \\
4 \mathrm{BM}-\mathrm{NR}(31)\end{array}$ \\
\hline Vascular adverse event on therapy, No (\%) & $6(10)$ & $3(10)$ & $2(13)$ & $1(8)$ \\
\hline MF transformation on therapy, no (\%) & $4(7)$ & $4(14)$ & 0 & 0 \\
\hline Duration of therapy, months (range) & $80(15-107)$ & $75(25-205)$ & $85(77-107)$ & $83(15-101)$ \\
\hline Total median follow-up, months (range) & $84(36-107)$ & $81(36-105)$ & $94(67-107)$ & $93(83-106)$ \\
\hline On study at last follow-up, no (\%) & $32(55)$ & $10(35)$ & $13(81)$ & $9(69)^{*}$ \\
\hline
\end{tabular}

$D X$ diagnosis, $H R$ hematologic response, $M R$ molecular response

* $\mathrm{p}<0.05$

a One patient was not evaluable for subsequent MR

b $8 / 13$ patients with BM-CR (61\%) achieved complete resolution of reticulin fibrosis (MF-0) after a median of 63 months on therapy (range $30-72$ ); the remaining 5 patients has decreased their BM fibrosis to MF 0/1 after a median of 48 months (range 30-69) on therapy 


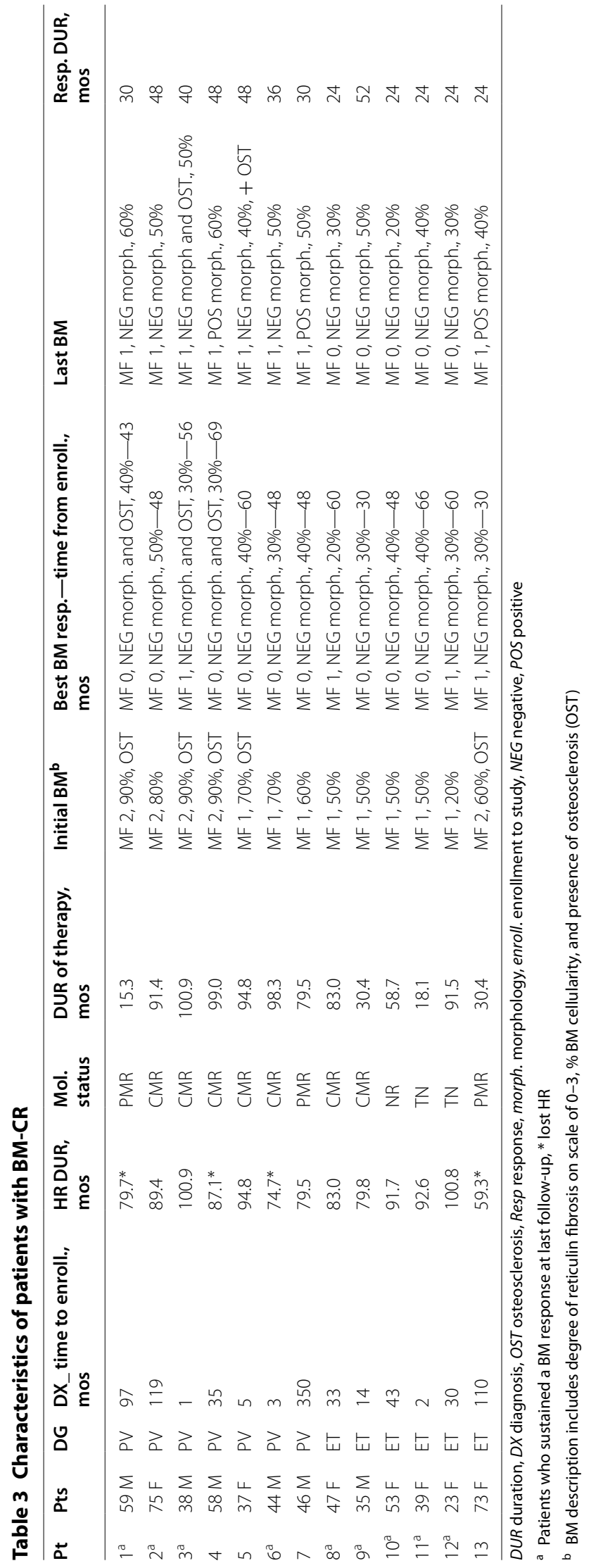


a

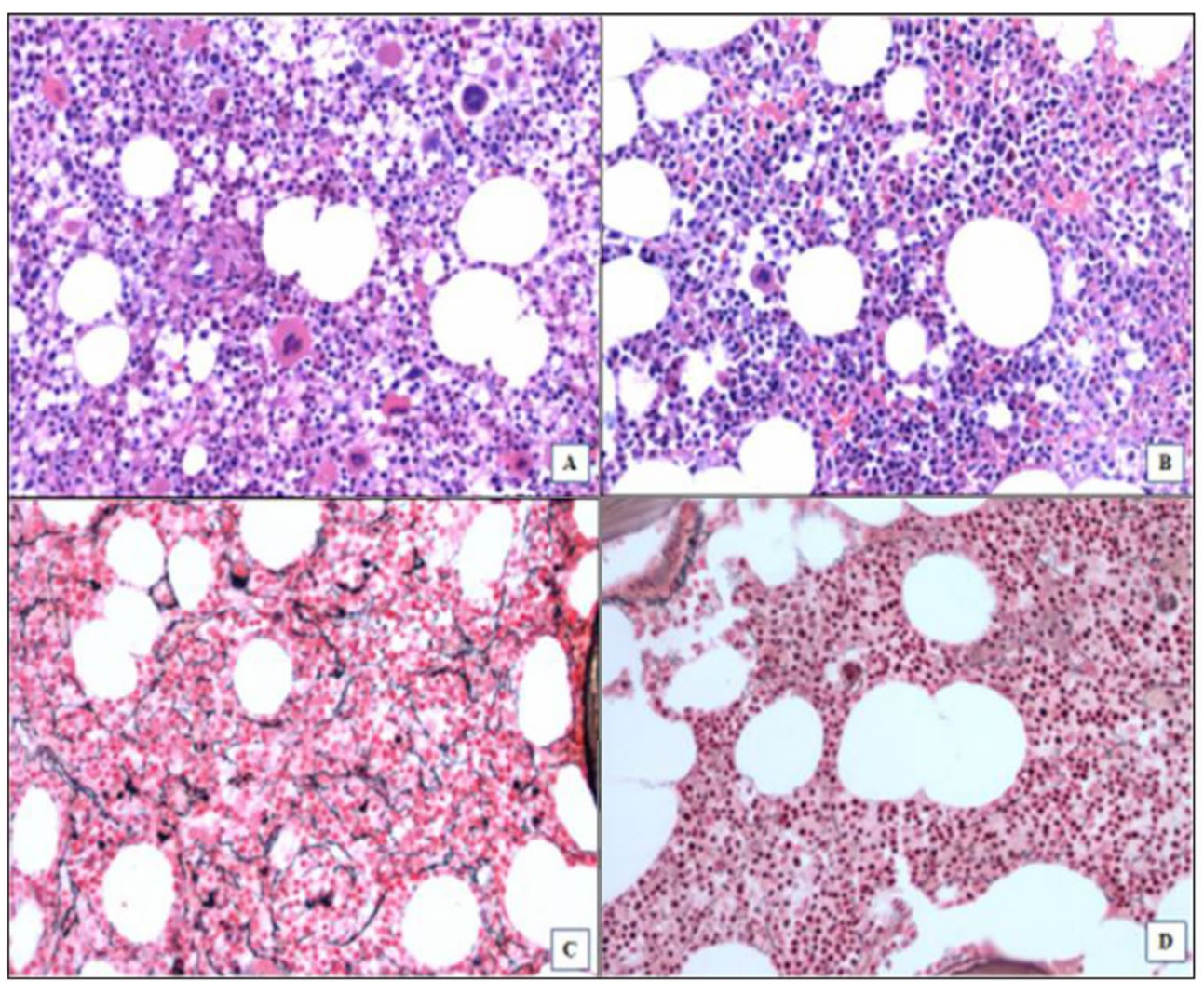

b

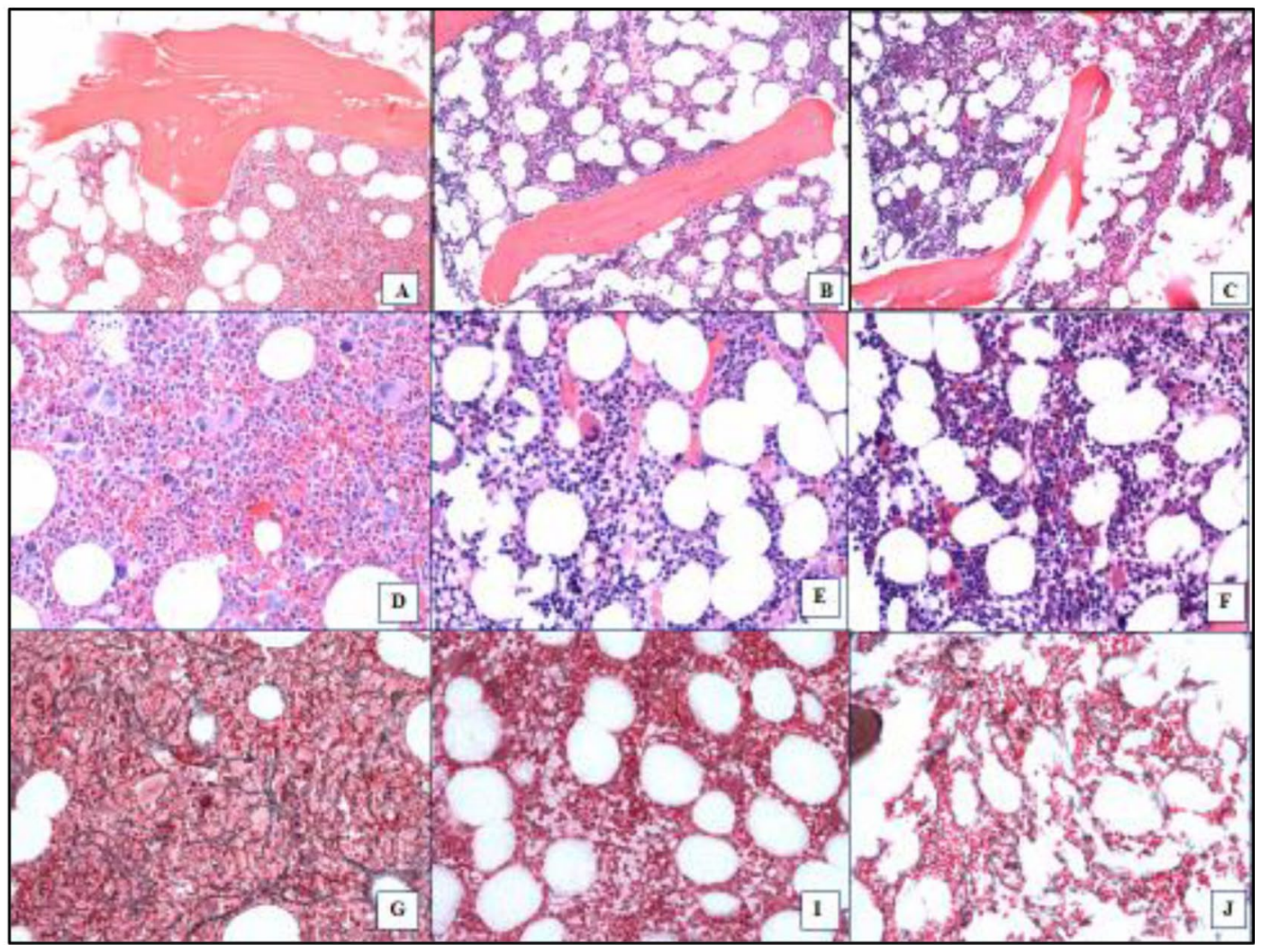


(See figure on previous page.)

Fig. 1 BM Response patterns observed in patients treated with PEG-INF-a-2a. a BM biopsy sections from a patient with ET who is still on therapy after $84+$ months with BM-CR and CMR that was achieved after 60 months on treatment (Pt 8, Fig. 3a). A, B Hematoxylin and eosin staining at (A) the start of therapy and (B) at BM-CR after 60 months on therapy. C, D reticulin staining showing BM fibrosis at (C) the start of therapy (MF-1/2) and $\mathbf{D}$ at the time of BM-CR (MF-0). b Patient with PV who is still on therapy after 96 + months who achieved a CHR, CMR and BM-CR but has only sustained a CMR (Pt 4, Fig. 3b). Loss of BM-CR occurred after 84 months and loss of CHR after 96 months. Hematoxylin and eosin staining showing osteosclerosis at (A) the start of therapy, B at the time of best response (60 months), and $\mathbf{C}$ at relapse after 84 months. D-F Hematoxylin and eosin staining showing BM cellularity and megakaryocyte morphology at (D) the start of therapy, $\mathbf{E}$ at the time of best response (60 months), and $\mathbf{F}$ at relapse (84 months). G, I, J Reticulin staining showing BM fibrosis at (G) the start of therapy (MF-2), I at the time of best response (MF-0), and $\mathbf{J}$ at relapse (MF-1). All images shown are magnified $\times 200$

molecular relapse during the entire follow-up, whilst it was observed in 2 of 3 patients with PMR. One patient who was actively treated on a study for 59 months, did not achieve any MR despite achieving a CHR and $\mathrm{BM}-\mathrm{CR}$, both currently ongoing despite $>40$ months off therapy.

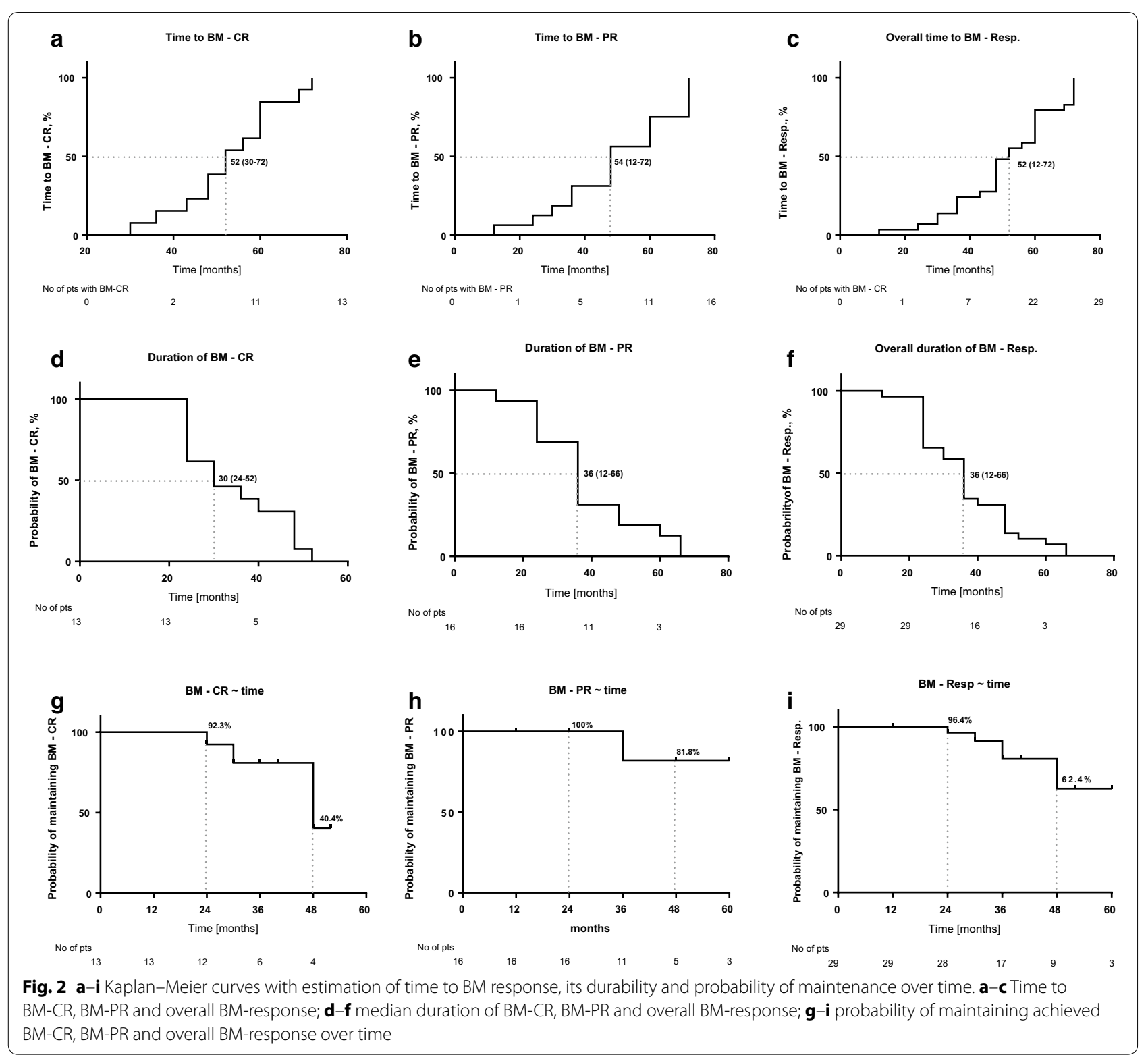




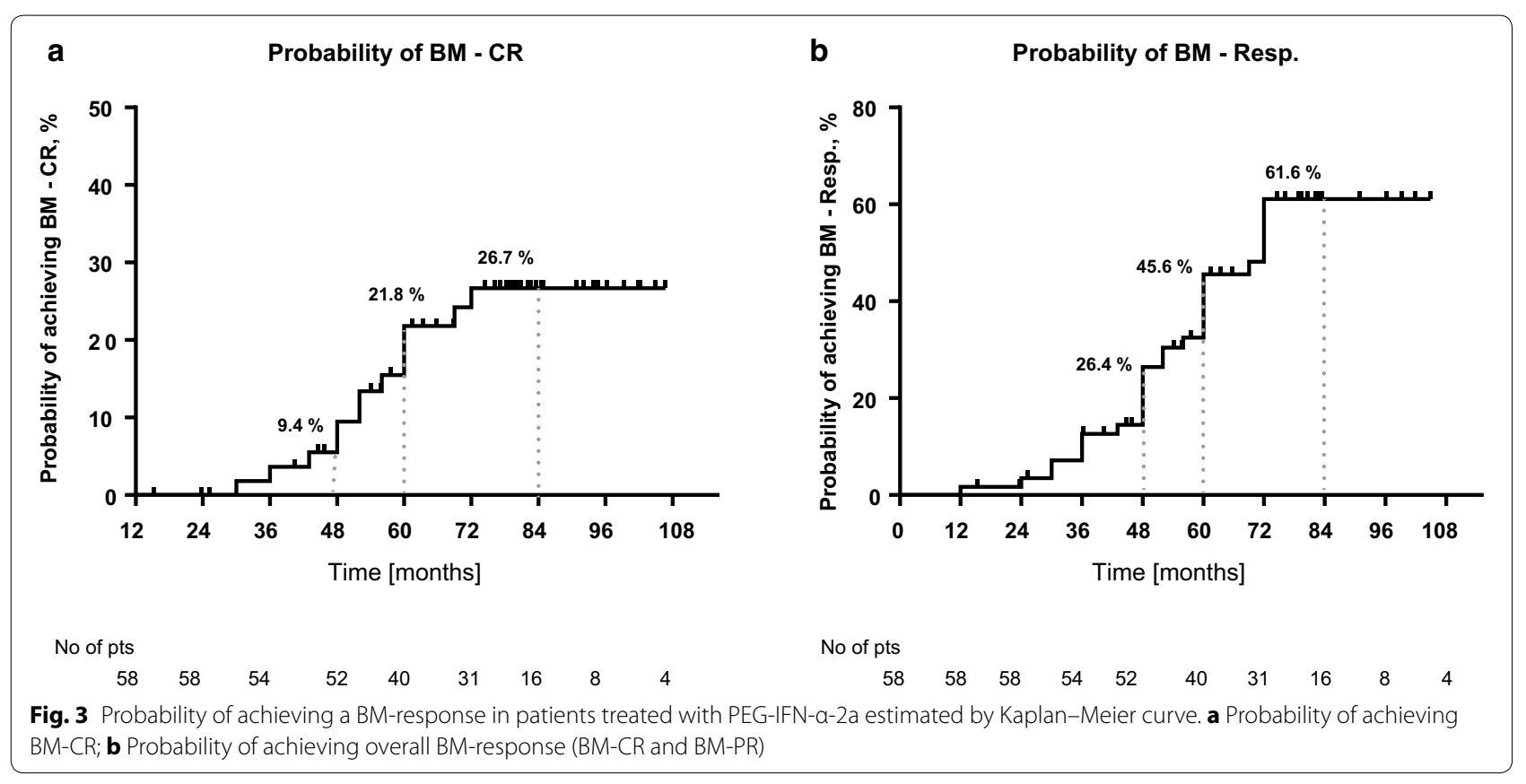

Four patients have lost their BM-CR (Fig. 6) after a median response duration of 39 months (range 30-48), and 3 of them were still on active therapy before they lost their BM-CR. Loss of BM response was accompanied by loss of CHR and PMR in 2 and 1 patient, respectively.

Given the fact that achievements of BM-CR allows for grade 1 fibrosis in the $\mathrm{BM}$, we have also evaluated the ability of PEG-IFN- $\alpha-2 a$ to revert BM reticulin fibrosis. Overall, 13 patients (22\%, BM-CR in 11, BM-PR in 2) have achieved complete resolution of initially present BM fibrosis (MF-2 or MF-1 to MF-0 in 3 and 10 patients, respectively), which was sustained in 5 patients at the time of last follow-up (all in BM-CR). Additional 18 patients (31\%, BM-CR in 2, BM-PR in 16) have experienced decrease in $\mathrm{BM}$ fibrosis by 1 grade (MF-2 to MF-1), which was sustained in 15 patients at the time of last follow-up (1 in BM-CR and 14 in BM-PR).

\section{Vascular complications}

Overall, 6 (10\%) patients experienced unprovoked vascular complications ( 5 thrombotic and one bleeding event): one while having BM-CR, 2 BM-PR, and 3 BM-NR. The incidence rate of major unprovoked thromboembolic event in enrolled patients during the entire study follow-up was 1.37 per 100 person years. One patient with BM-CR experienced pulmonary embolism after being treated for 14 months, while being in CHR for past 6 months. The patient continued on therapy with PEGIFN- $\alpha-2 \mathrm{a}$ after the event for another 80 months, was able to achieve CMR (JAK2 allele burden at the time of embolism was 75\%), and BM-CR, which were all sustained at the time of last follow-up, when the patient discontinued treatment due to financial reasons. Both patients with BM-PR developed vascular complications (femoral arterial thrombosis and hemorrhagic stroke in one each) after therapy for 24 and 36 months, respectively; one patient was in CHR and the other one has never achieved HR. Both continued on therapy after the vascular event occurred for additional 42 and 60 months, respectively, and discontinued the treatment for financial reasons.

\section{Disease progression}

Progression to overt MF and AML occurred in 5 patients (9\%; $4 \mathrm{MF}$ and $1 \mathrm{AML}$ ), and none of these patients had a $\mathrm{BM}$ response. One patient who progressed to AML was holding PEG-IFN- $\alpha$-2a for 6 months before transformation for unrelated reason (knee replacement surgery). None of the 4 patients were in CHR at the time of progression to MF, and all have discontinued the therapy afterwards.

\section{Patients after therapy discontinuation}

Among patients with a BM response who discontinued therapy, 6 continued to have BM biopsies performed, with a median of 4 samples per patient (range 3-6). Four stopped therapy while in BM-CR and 2 while in BM-PR.

Two patients (\#1 and \#11, Figs. 4, 5), who had achieved a BM-PR at the time of treatment discontinuation, continued to show improvements in their BM despite being 

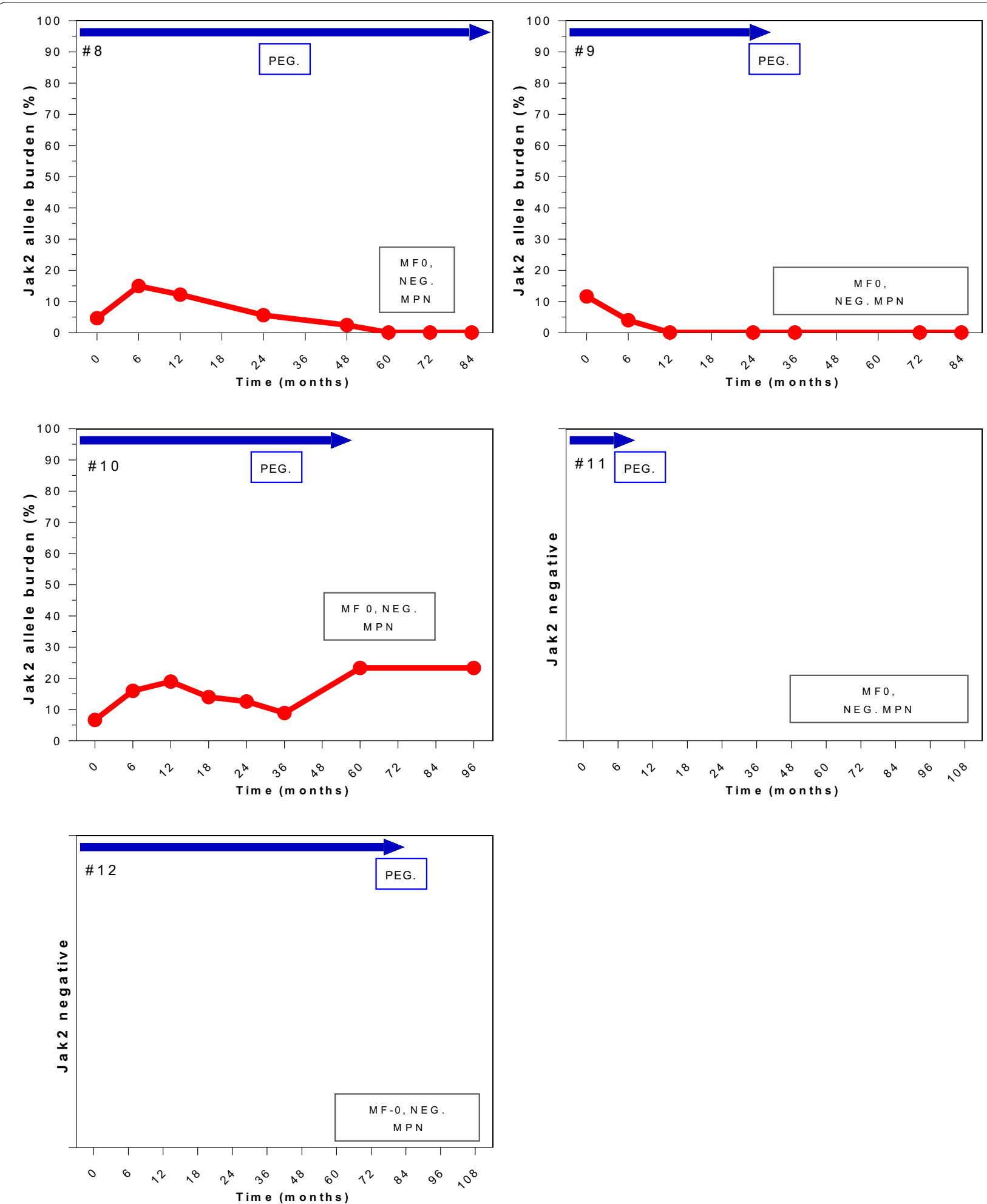

Fig. 4 Correlation of molecular and morphological responses over time in selected patients. Patients with a sustained BM-CR (MF-0). Blue arrows indicate time on active therapy. The number in the top left upper corner represents the patient \# from Table 2. The red line indicates the JAK2 allele burden over time (missing in TN patients). The box in the bottom right corner shows the change in BM and response, NEG MPN = no signs of ET or $\mathrm{PV}$, normal BM. The gray square represents the time of loss of HR (shown only in patients who lost their HR) 

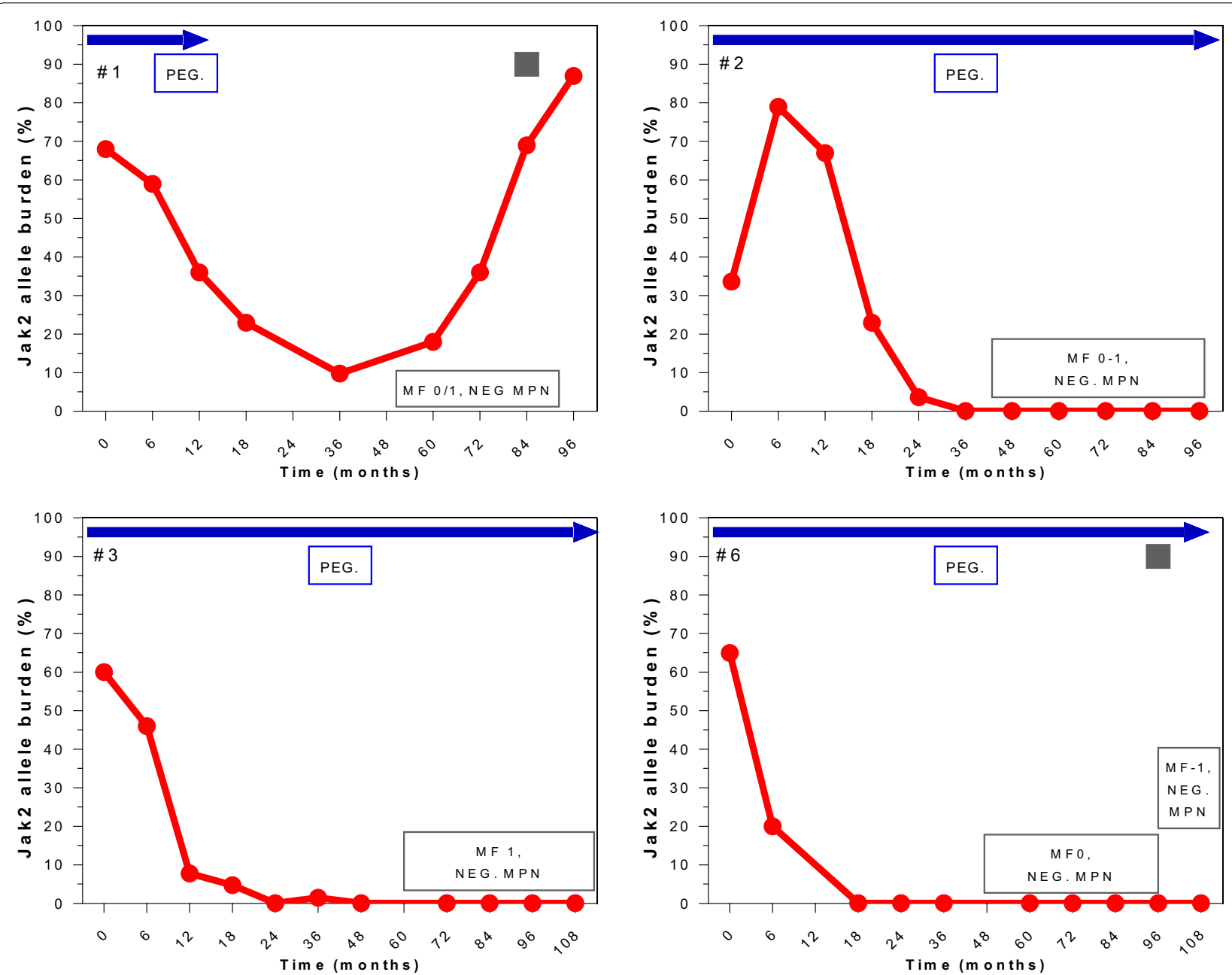

Fig. 5 Correlation of molecular and morphological responses over time in selected patients. Patients with a sustained BM-CR with mild reticulin fibrosis (MF 0/1). The number in the top left upper corner represents the patient \# from Table 2. The red line indicates the JAK2 allele burden over time. The box in the bottom right corner shows the change in BM and response, NEG MPN = no signs of ET or PV, normal BM. The gray square represents the time of loss of HR (shown only in patients who lost their HR)

off therapy. Remarkably, both achieved a BM-CR after being off therapy for 32 and 36 months, and maintained their BM-CR for 30 and 24 months, still off therapy, respectively. One of these patients (\#1, Fig. 5) has lost his CHR and best MR while still remaining in BM-CR; the other one continues to be in CHR (MR not evaluable for JAK2 negativity). Among those 4 patients who stopped therapy at the time of BM-CR (\#9, \#10, \#12, and \#13, Fig. 4), 3 remain in sustained BM-CR. One of them has lost his best MR (\#10, Fig. 4), one remains in CMR (\#9, Fig. 4) and one is JAK2 negative (\#12, Fig. 4). All three patients maintain their initial CHR. Only one patient has lost his BM-CR after being off therapy for 24 months, he has also lost his CHR and best MR (\#13, Fig. 6).
At present, therefore, 5 of 6 evaluable patients are in BM-CR after stopping therapy, and are maintaining their BM-CR for a median time of follow-up after therapy discontinuation of 48 months (range 32-68).

\section{Discussion}

We report on histomorphological BM responses in 58 patients with ET/PV treated with PEG-IFN- $\alpha-2 a$ in a prospective, single-center, phase 2 clinical trial after a therapy for a median of 84 months. Our current research substantiates existing data showing that long-term therapy with PEG-IFN- $\alpha-2 \mathrm{a}$ is not only able to induce CHR and CMR, but could also lead to complete normalization of BM morphology in a subset of patients. Moreover, PEG-IFN- $\alpha-2 \mathrm{a}$ was capable to completely reverse 

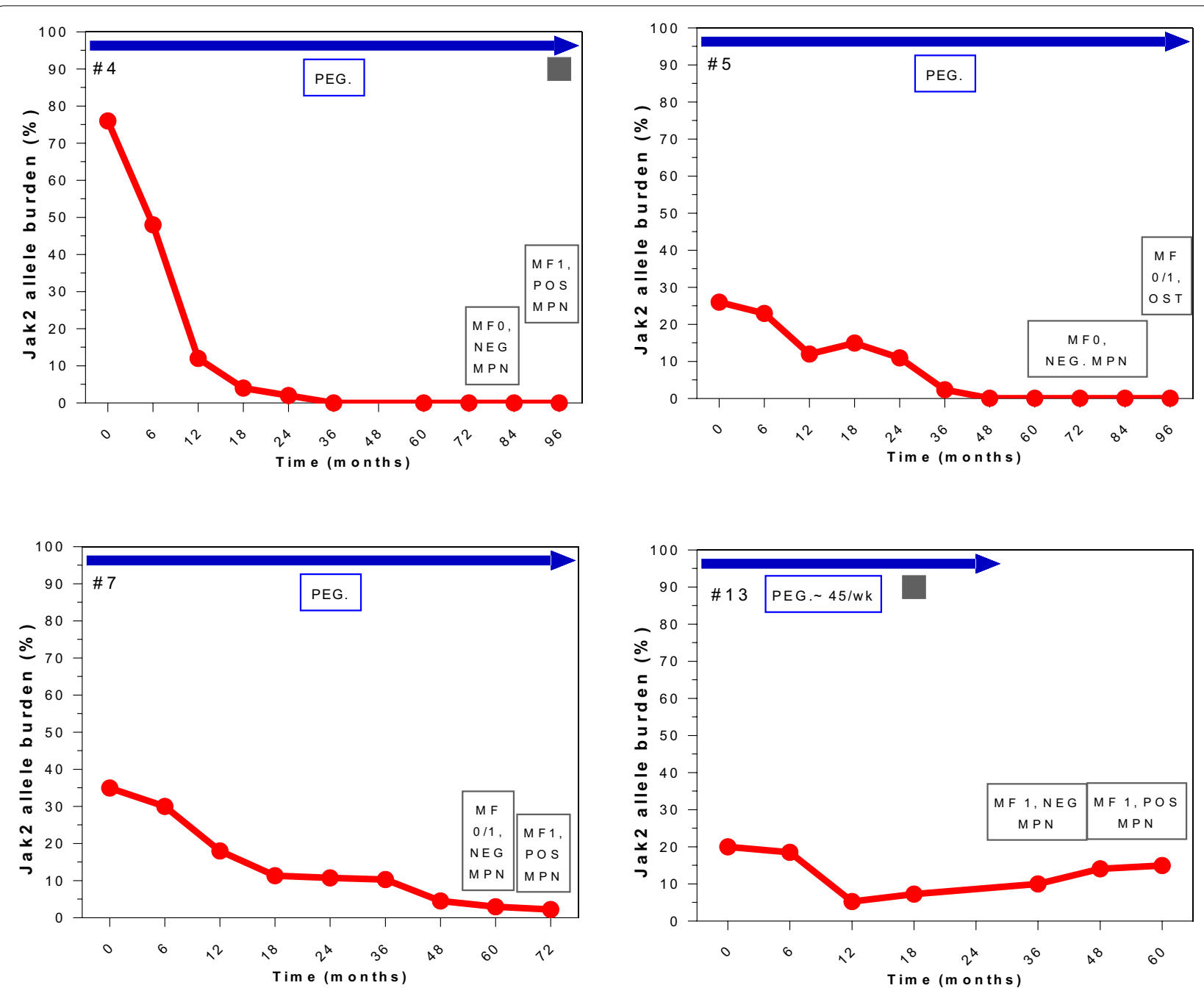

Fig. 6 Correlation of molecular and morphological responses over time in selected patients. Patients with BM-CR (MF-0/1, MF-1) and subsequent relapse. Blue arrows indicate time on active therapy. The number in the top left upper corner represents the patient \# from Table 2. The red line indicates the JAK2 allele burden over time. The box in the bottom right corner shows the change in BM and response, NEG MPN = no signs of ET or $P V$, normal BM. The gray square represents the time of loss of HR (shown only in patients who lost their HR)

BM fibrosis in up to $22 \%$ of patients, which is higher than previously observed by other investigators $[23,26]$. BM responses were achieved after median time on therapy of 48 months, confirming the observation that a long treatment duration is necessary to achieve such a response. Our study also reports a few novel observations. In contrast to previously published results [21], we have noticed significant reduction in BM cellularity in patients with PV who achieved CMR. Patients with BM-CR seemed to derive the most benefits from the therapy with longest duration of initial responses (CHR and CMR), higher rates of CMR, and the fewest disease-related complications. Importantly, none of the patients who had achieved BM-CR progressed to overt MF, and majority of patients who had lost their initial BM-CR have maintained their hematologic and molecular responses. BM-CR was durable, as $69 \%$ of them were sustained at the time of last follow-up. Furthermore, 5 of 6 (83\%) patients who continued to have BM evaluations after treatment discontinuation, have maintained their BM-CR after a median duration off therapy of 48 months. However, 2 of them have lost their best MR at the time of last follow-up, and one patients has lost his hematologic response.

Despite the fact that patients with BM-CR appeared to benefit from the therapy the most, we could not identify any predictive markers of or a correlation between the achievement of hematologic/molecular responses and $\mathrm{BM}$ response. Achievement of $\mathrm{BM}$ response or its durability was not essential for obtaining hematological or molecular response or clinical benefit (i.e., remain 
symptom free with no organomegaly or thrombotic event), and therefore can't be considered as major point for a treatment decision making in a clinical practice.

One would expect that loss of HR or MR will be accompanied by the loss of BM response; and it might happen in these patients over time. However, in spite of the loss of $H R$ and MR, these patients remain symptoms free without vascular complications while off any active therapy. The fact that their BM is not showing signs of MPN, yet their laboratory parameters (hematologic or molecular) indicate the presence of disease, further echoes our limited understanding of the dynamics of these responses, and its clinical significance. Moreover, it also introduces a question about the definition of useful objective and clinically meaningful response in these patients, as simply relying on the hematologic parameters in a patient with no clinical signs of disease may not be accurate, especially after or during therapy with a biological agents such as interferon. Hopefully, with longer follow-up and better "response" assessment tools, e.g. deep genome sequencing, we will gain better insight into this dilemma.

Notwithstanding its unclear clinical meaning, our results show the ability of PEG-IFN- $\alpha-2 \mathrm{a}$ to reverse BM fibrosis, confirming its significant biological activity. Furthermore, the observation that these responses could be sustained even after treatment discontinuation, reinforces its strong immunomodulatory effects with a potential for future treatment approaches. Combining PEG-IFN- $\alpha$-2a with other therapies that have been shown to possibly reverse or reduce BM fibrosis in patients with MF, should be explored in clinical trials.

However, due to the fact that PEG-IFN- $\alpha-2 a$ is capable of controlling the disease only for a limited time and progression (loss of achieved hematologic and molecular response, worsening of marrow fibrosis and even transformation to overt MF or AML) could still occur on therapy without any known biological correlations or predictive markers, we need to improve our ability to identify patients who would benefit the most from the treatment.

Major limitation of our trial is that despite its prospective character, morphologic evaluation was not predefined as one of the study endpoints, and therefore patients were evaluated retrospectively based on availability of BM samples during therapy, directed in part by them staying on therapy/responding, which could cause subjective bias.

In summary, our data confirmed the ability of PEGIFN- $\alpha-2 a$ to induce normalization of BM morphology in a subgroup of ET and PV patients. Although we have noticed that patients with a BM-CR may benefit from the therapy the most, with the longest disease control, no predictive markers to guide clinical decision were found. The significance of these findings as well as the prognostic value of BM response in patients with PV and ET remains incompletely understood. Our findings further highlight the need for more elaborate approach in assessing biological effects of interferon-alfa in ET and $\mathrm{PV}$, to help us understand the heterogeneity and dynamics of clinical, hematologic, molecular and morphologic responses and their significance during long-term therapy with PEG-IFN- $\alpha-2 \mathrm{a}$.

\section{Conclusions}

PEG-IFN- $\alpha-2 a$ is capable to induce complete BM responses in a subset of ET and PV patients, but its correlation with durable clinically relevant treatment benefit warrants further investigation.

\section{Abbreviations \\ AML: acute myeloid leukemia; BM: bone marrow; BM-CR: bone marrow complete response; BM-PR: bone marrow partial response; BM-NR: bone mar- row no response; ET: essential thrombocythemia; HR: hematologic response; IWG-MRT: International Working Group-Myeloproliferative Neoplasms Research and Treatment; MR: molecular response; CMR: molecular response, complete [undetectable JAK2V617F allele burden]; PR: molecular response, partial [50\% reduction in JAK2V617F allele burden]; mMR: molecular response, minor [20-49\% reduction in JAK2V617F allele burden]; MF: myelofibrosis; MPN: myeloproliferative neoplasms; PEG-IFN-a-2a: pegylated interferon alpha-2a; PV: polycythemia vera.}

\section{Authors' contributions}

SV and HK designed and coordinated the original clinical trial. LM and SV analyzed the data. CY and CBR performed morphologic analysis. LM, KJN, and SV wrote the manuscript. JC, MC, GB, HK, SV, LM enrolled patients and did the research. All authors participated in the discussion, have reviewed and approved. All authors read and approved the final manuscript.

\section{Author details}

${ }^{1}$ Department of Leukemia, The University of Texas MD Anderson Cancer Center, 1515 Holcombe Blvd., Unit 428, Houston, TX 77030, USA. ${ }^{2}$ Department of Hematopathology, The University of Texas MD Anderson Cancer Center, Houston, TX 77030, USA

\section{Acknowledgements}

This research is supported in part by the MD Anderson Cancer Center Support Grant P30 CA016672 from the US National Cancer Institute.

\section{Competing interests}

The authors declare that they have no competing interests.

\section{Availability of data}

The datasets used and analyzed during the current study as available from the corresponding author on reasonable request, however restrictions apply to the availability of the data under the license for this study.

\section{Consent for publication}

Not applicable.

\section{Ethics approval and consent to participate}

This study was approved by the Institutional Review Board at MD Anderson Cancer Center and all patients signed an informed consent document.

\section{Funding}

This study was funded in part through the MD Anderson Cancer Center Support Grant P30 CA016672 from the National Cancer Institute. 


\section{Publisher's Note}

Springer Nature remains neutral with regard to jurisdictional claims in published maps and institutional affiliations.

Received: 26 September 2017 Accepted: 27 October 2017 Published online: 09 November 2017

\section{References}

1. Marchioli R, Finazzi G, Landolfi R, Kutti J, Gisslinger H, Patrono C, Marilus R, Villegas A, Tognoni G, Barbui T. Vascular and neoplastic risk in a large cohort of patients with polycythemia vera. J Clin Oncol. 2005;23(10):2224-32.

2. Wolanskyj AP, Schwager SM, McClure RF, Larson DR, Tefferi A. Essential thrombocythemia beyond the first decade: life expectancy, longterm complication rates, and prognostic factors. Mayo Clin Proc. 2006;81(2):159-66.

3. Fruchtman SM, Mack K, Kaplan ME, Peterson P, Berk PD, Wasserman LR. From efficacy to safety: a Polycythemia Vera Study group report on hydroxyurea in patients with polycythemia vera. Semin Hematol. 1997;34(1):17-23

4. Silver RT. Recombinant interferon-alpha for treatment of polycythaemia vera. Lancet. 1988;2(8607):403.

5. Kiladjian JJ, Chomienne C, Fenaux P. Interferon-alpha therapy in bcr-ablnegative myeloproliferative neoplasms. Leukemia. 2008;22(11):1990-8.

6. Quintas-Cardama A, Kantarjian H, Manshouri T, Luthra R, Estrov Z, Pierce S, Richie MA, Borthakur G, Konopleva M, Cortes J, et al. Pegylated interferon alfa-2a yields high rates of hematologic and molecular response in patients with advanced essential thrombocythemia and polycythemia vera. J Clin Oncol. 2009;27(32):5418-24.

7. Kiladjian JJ, Cassinat B, Chevret S, Turlure P, Cambier N, Roussel M, Bellucci S, Grandchamp B, Chomienne C, Fenaux P. Pegylated interferon-alfa-2a induces complete hematologic and molecular responses with low toxicity in polycythemia vera. Blood. 2008;112(8):3065-72.

8. Masarova L, Patel KP, Newberry KJ, Cortes J, Borthakur G, Konopleva M, Estrov Z, Kantarjian H, Verstovsek S. Pegylated interferon alfa-2a in patients with essential thrombocythaemia or polycythaemia vera: a post hoc, median 83 month follow-up of an open-label, phase 2 trial. Lancet Haematol. 2017;4:e165-75.

9. Pizzi M, Silver RT, Barel A, Orazi A. Recombinant interferon-alpha in myelofibrosis reduces bone marrow fibrosis, improves its morphology and is associated with clinical response. Mod Pathol. 2015:28(10):1315-23.

10. Silver RT, Vandris K, Goldman JJ. Recombinant interferon-alpha may retard progression of early primary myelofibrosis: a preliminary report. Blood. 2011;117(24):6669-72.

11. Arber DA, Orazi A, Hasserjian R, Thiele J, Borowitz MJ, Le Beau MM, Bloomfield CD, Cazzola M, Vardiman JW. The 2016 revision to the World Health Organization classification of myeloid neoplasms and acute leukemia. Blood. 2016;127(20):2391-405

12. Barosi G, Mesa R, Finazzi G, Harrison C, Kiladjian JJ, Lengfelder E, McMullin MF, Passamonti F, Vannucchi AM, Besses C, et al. Revised response criteria for polycythemia vera and essential thrombocythemia: an ELN and IWGMRT consensus project. Blood. 2013;121(23):4778-81.

13. Barbui T, Thiele J, Passamonti F, Rumi E, Boveri E, Randi ML, Bertozzi I, Marino F, Vannucchi AM, Pieri L, et al. Initial bone marrow reticulin fibrosis in polycythemia vera exerts an impact on clinical outcome. Blood. 2012;119(10):2239-41.

14. Abdulkarim K, Ridell B, Johansson P, Kutti J, Safai-Kutti S, Andreasson B. The impact of peripheral blood values and bone marrow findings on prognosis for patients with essential thrombocythemia and polycythemia vera. Eur J Haematol. 2011;86(2):148-55.
15. Campbell PJ, Bareford D, Erber WN, Wilkins BS, Wright P, Buck G, Wheatley K, Harrison CN, Green AR. Reticulin accumulation in essential thrombocythemia: prognostic significance and relationship to therapy. J Clin Oncol. 2009;27(18):2991-9.

16. Hauck G, Jonigk D, Gohring G, Kreipe H, Hussein K. Myelofibrosis in Philadelphia chromosome-negative myeloproliferative neoplasms is associated with aberrant karyotypes. Cancer Genet. 2013;206(4):116-23.

17. Barraco D, Cerquozzi S, Hanson CA, Ketterling RP, Pardanani A, Gangat $\mathrm{N}$, Tefferi A. Prognostic impact of bone marrow fibrosis in polycythemia vera: validation of the IWG-MRT study and additional observations. Blood Cancer J. 2017:7(3):e538.

18. Essers MA, Offner S, Blanco-Bose WE, Waibler Z, Kalinke U, Duchosal MA, Trumpp A. IFNalpha activates dormant haematopoietic stem cells in vivo. Nature. 2009;458(7240):904-8.

19. Chott A, Gisslinger H, Thiele J, Fritz E, Linkesch W, Radaszkiewicz T, Ludwig H. Interferon-alpha-induced morphological changes of megakaryocytes: a histomorphometrical study on bone marrow biopsies in chronic myeloproliferative disorders with excessive thrombocytosis. Br J Haematol. 1990;74(1):10-6.

20. Hasselbalch HC, Larsen TS, Riley CH, Jensen MK, Kiladjian JJ. Interferonalpha in the treatment of Philadelphia-negative chronic myeloproliferative neoplasms. Status and perspectives. Curr Drug Targets. 2011;12(3):392-419.

21. Margolskee EB, Krichevsky S, Orazi A, Silver RT. Evaluation of bone marrow morphology is essential for assessing disease status in recombinant interferon alpha-treated polycythemia vera patients. Haematologica. 2016:102:e97-9.

22. Larsen TS, Bjerrum OW, Pallisgaard N, Andersen MT, Moller MB, Hasselbalch HC. Sustained major molecular response on interferon alpha-2b in two patients with polycythemia vera. Ann Hematol. 2008:87(10):847-50.

23. Utke Rank C, Weis Bjerrum O, Larsen TS, Kjaer L, de Stricker K, Riley CH, Hasselbalch HC. Minimal residual disease after long-term interferonalpha2 treatment: a report on hematological, molecular and histomorphological response patterns in 10 patients with essential thrombocythemia and polycythemia vera. Leuk Lymphoma. 2015;18:1-7.

24. Quintas-Cardama A, Abdel-Wahab O, Manshouri T, Kilpivaara O, Cortes J, Roupie AL, Zhang SJ, Harris D, Estrov Z, Kantarjian H, et al. Molecular analysis of patients with polycythemia vera or essential thrombocythemia receiving pegylated interferon alpha-2a. Blood. 2013;122(6):893-901.

25. Thiele J, Kvasnicka HM, Facchetti F, Franco V, van der Walt J, Orazi A. European consensus on grading bone marrow fibrosis and assessment of cellularity. Haematologica. 2005;90(8):1128-32.

26. Larsen TS, Møller MB, de Stricker K, Nørgaard P, Samuelsson J, Marcher C, Andersen MT, Bjerrum OW, Hasselbalch HC. Minimal residual disease and normalization of the bone marrow after long-term treatment with alpha-interferon $2 \mathrm{~b}$ in polycythemia vera. A report on molecular response patterns in seven patients in sustained complete hematological remission. Hematology. 2009;14(6):331-4.

\section{Submit your next manuscript to BioMed Central and we will help you at every step:}

- We accept pre-submission inquiries

- Our selector tool helps you to find the most relevant journal

- We provide round the clock customer support

- Convenient online submission

- Thorough peer review

- Inclusion in PubMed and all major indexing services

- Maximum visibility for your research

Submit your manuscript at www.biomedcentral com/submit 